\title{
Continuidade das ações em saúde na atenção ambulatorial especializada durante a pandemia pela Covid-19
}

\author{
The continuity of health actions in specialized am- \\ bulatory care during the Covid-19 pandemic
}

\begin{abstract}
Jéssica Oliveira de Lima ${ }^{1}$, Maria Goretti David Lopes ${ }^{2}$, Carmen Cristina Moura dos Santos ${ }^{3}$
1. ORCID: https://orcid.org/0000-0003-1264-2193. Enfermeira especialista em Saúde da Família (Faculdades Pequeno Príncipe - 2016) e em Gestão da Saúde (Universidade Federal do Paraná - 2019). Secretaria de Estado da Saúde do Paraná (Curitiba, Paraná, Brasil). E-mail: jessica. oliveira@sesa.pr.gov.br.

2. ORCID: https://orcid.org/0000-0001-7473-6342. Enfermeira especialista em Planejamento e Desenvolvimento de Recursos Humanos para a Saúde. Diretora de Atenção e Vigilância em Saúde da Secretaria de Estado da Saúde do Paraná (Curitiba, Paraná, Brasil).

3. ORCID: https://orcid.org/0000-0003-3162-8346. Enfermeira especialista em Gestão da Clínica nos Hospitais do SUS. Coordenadora da Organização da Rede de Cuidados em Saúde da Secre-
\end{abstract} taria de Estado da Saúde do Paraná (Curitiba, Paraná, Brasil).

CONTATO: Jéssica Oliveira de Lima | Rua Piquiri, no 170, Rebouças, Curitiba. Telefone: (41) 98876-1001 | E-mail: jeholima@gmail.com | jessica.oliveira@sesa.pr.gov.br

RESUMO Os sistemas de saúde precisaram construir planos de ações e respostas rápidas para o enfrentamento da Covid-19; no Brasil, os arranjos organizativos do Sistema Único de Saúde foram fundamentais para operacionalizar as estratégias traçadas. A organização dos serviços de saúde em níveis de complexidade, interligados por meio da Rede de Atenção à Saúde, permitiu que a Secretaria do Estado da Saúde do Paraná traçasse, precocemente, prioridades para esse enfrentamento. O objetivo deste relato de experiência é evidenciar as orientações destinadas aos serviços ambulatoriais especializados do Estado, com enfoque no seguimento dos usuários com condições crônicas, evitando instabilidade e agravamento da saúde. Foram construídas, pelas equipes técnicas, orientações que fomentaram os processos de reorganização da Rede. Destacamos as Resoluções, as Notas Orientativas e o Plano de 
Contingência Estadual, que embasaram a continuidade das ações em saúde, de forma resolutiva e segura, auxiliando os gestores e profissionais de saúde no direcionamento dos esforços.

DESCRITORES: Pandemia. Coronavírus. Comorbidade. Atenção à Saúde.

ABSTRACT Health systems needed to build action plans and quick responses to cope with Covid-19; in Brazil, the organizational arrangements of the Unified Health System were fundamental to operationalize the outlined strategies. The organization of health services in levels of complexity, interconnected through the Health Care Network, allowed the Paraná State Health Secretary to set priorities for this confrontation at an early stage. The purpose of this experience report is to highlight the guidelines for specialized outpatient services in the State, with a focus on monitoring users with chronic conditions, avoiding their instability and worsening health. Guidelines were developed by technical teams that fostered the Network's reorganization processes. We highlight the Resolutions, Guidance Notes, and the State Contingency Plan, which supported the continuity of health actions, resolutely and safely, assisting managers and health professionals in directing efforts.

DESCRIPTORS: Pandemic. Coronavirus. Comorbidity. Health Care.

\section{INTRODUÇÃO}

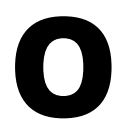

enfrentamento à pandemia por Covid-19 se tornou um desafio e uma corrida contra o tempo para os sistemas de saúde em todo o mundo. A doença exige resposta rápida das autoridades, além de logística, insumos e recursos humanos suficientes para garantir o atendimento à população acometida pelo SARS-CoV-2 $2^{1-2}$.

O Brasil conta com uma organização diferenciada da assistência à saúde, e possui o Sistema Único de Saúde (SUS), estabelecido pela Constituição Federal de 1988, que destaca a saúde como um direito de todos os cidadãos brasileiros, e um dever do Estado. O SUS é operacionalizado mediante políticas públicas e sociais que contemplem a integralidade do indivíduo, por meio de princípios e diretrizes que o fundamentam ${ }^{3}$. 
Por intermédio dos arranjos organizativos que o SUS construiu com o passar dos anos, entre eles as Redes de Atenção à Saúde (RAS), foi possível garantir, em meio a grandes dificuldades de financiamento, a regionalização e a hierarquização, a universalidade, a equidade e a integralidade, princípios previstos desde a sua constituição ${ }^{4}$.

Para o enfrentamento da pandemia no Brasil, a organização em RAS tem se mostrado estratégia e efetiva na superação dos efeitos deletérios dessa situação de emergência pública. Reforçar o papel ordenador e resolutivo da Atenção Primária à Saúde (APS) é primordial nesse momento, assim como garantir que os usuários com condições crônicas sejam assistidos pelas equipes Atenção Ambulatorial Especializada (AAE), sem interrupção e prejuízo do acompanhamento previsto no plano de cuidados multiprofissional ${ }^{3-4}$.

As preocupações e os esforços iniciais das autoridades sanitárias, no âmbito mundial, estão relacionados à propagação viral. Medidas preventivas foram recomendadas, com destaque ao distanciamento social, uso universal de máscaras, incentivo à higienização correta das mãos e prática de etiqueta respiratória. Paralelamente, foram organizadas estratégias direcionadas à promoção do isolamento social, organização dos processos de trabalho e das atividades relativas ao comércio e educação, juntamente ao fortalecimento dos sistemas de saúde para o enfrentamento da pandemia.

Estimativas sugerem que $81 \%$ das pessoas acometidas pela Covid-19 são assintomáticas ou apresentam sintomas leves, e condições clínicas para manejo na APS. Entretanto, 14\% dos doentes necessitarão de internamento hospitalar, com demanda de 5\% para ocupação de leitos de UTI, reiterando a importância da Rede de Urgência Emergência (RUE), e da Rede Hospitalar nesse processo organizativo ${ }^{2}$.

Com o surgimento da primeira onda da doença no Brasil, evidenciada pelo aumento do número de casos confirmados e de óbitos, foi necessário organizar o sistema de saúde, a fim de garantir atendimento àqueles que necessitassem 
de suporte hospitalar ${ }^{5}$. Contudo, a rápida propagação, com consequente aumento da procura por serviços de emergência e hospitalares insuflou, em um curto espaço de tempo, a capacidade e a ocupação dos leitos de média e alta complexidade. Isso contribuiu para a ocorrência da segunda onda da doença, evidenciada pela falta de equipamentos e insumos, além do desgaste dos recursos humanos em saúde 5 .

Enfrentando um dos momentos mais críticos em nível de organização das RAS já se vislumbra, em um curto espaço de tempo, a formação de uma nova onda que poderá sobrecarregar, de outra forma, os serviços de saúde, ocasionada pelo agravamento de condições crônicas não monitoradas e negligenciadas, ou diagnosticadas tardiamente ${ }^{5-6}$. É na perspectiva da continuidade das ações dentro das RAS, de forma segura, que essa nova onda poderá ser contida. Os olhares dos profissionais de saúde e gestores precisam contemplar as demais condições que afetam a população, de forma concomitante à pandemia ${ }^{2-5}$.

Isto posto, ao se observar as características e o comportamento da nova doença, identificou-se a necessidade de garantir que os demais níveis de atenção da RAS continuem a exercer o seu papel, dentro das competências atribuídas a cada um desses níveis. Nesse contexto, a Secretaria do Estado da Saúde do Paraná ( $S E S A=P R)$, dentre as diversas estratégias traçadas para o enfrentamento à pandemia, estabeleceu mecanismos para o fortalecimento da RAS e das linhas de cuidado prioritárias. Respostas rápidas foram tomadas e ações iniciais, voltadas à organização da RUE e suporte hospitalar específico, foram primordiais. A provisão de equipamentos, insumos e recursos humanos suficientes também fizeram parte dessa estratégia, a fim de evitar insuficiências.

Entre as estratégias, destaca-se a organização e as orientações destinadas aos serviços ambulatoriais especializados no Paraná, tema deste relato de experiência, bem como, a preocupação da gestão em garantir o seguimento dos usuários com condições crônicas, evitando instabilidade e seu agravamento. 


\section{MÉTODO}

Trata-se de relato de experiência que apresenta, descreve e discute a organização da Atenção Ambulatorial Especializada (AAE) no Estado do Paraná, para o enfrentamento da pandemia por Covid-19, e as estratégias desenhadas pelas equipes técnicas da Diretoria de Atenção e Vigilância em Saúde, em conjunto com outras diretorias da SESA-PR. O período de abrangência deste relato é de março a junho de 2020, e foi embasado no Plano de Contingência do Paraná para enfrentamento da Covid-19, nas resoluções e notas orientativas publicadas na página online da Secretaria. A construção e atualização dessas orientações ocorreram desde o período anterior ao registro de casos no Paraná/Brasil, e permanecem durante a pandemia.

O relato foi estruturado de forma descritiva, com concomitante discussão dos conteúdos e intercessão da literatura. Considerando tratar-se de documentos de acesso público, disponíveis no endereço eletrônico da SESA-PR, e em consonância com a Resolução do Conselho Nacional de Saúde n 510, de 7 de abril de 2016, esta publicação não foi submetida à apreciação por Comitê de Ética em Pesquisa.

\section{RESULTADOS E DISCUSSÃO}

Na RAS estão presentes serviços de diferentes densidades tecnológicas, vinculados a único objetivo, que é proporcionar atenção resolutiva, contínua e integral à população sob sua responsabilidade, através da cooperação e interdependência entre os níveis de atenção4 .

O acesso aos serviços disponíveis pela população, nessa Rede, ocorre de forma hierarquizada, regionalizada e organizada, sendo a APS a porta de entrada e a garantia de atendimento aos demais níveis. De forma complementar e sequencial ao atendimento prestado pela APS, destaca-se a AAE no arranjo organizativo do SUS, que desenvolve um conjunto de ações, práticas e técnicas 
assistenciais que transcendem a APS e incorporam maior densidade tecnológica, chamadas de tecnologias especializadas ${ }^{7}$.

A AAE é delimitada pela assistência sistematizada de profissionais especializados em determinado ramo da saúde, acompanhada de recursos tecnológicos de apoio diagnóstico e terapêutico. No Paraná, essa assistência é disponibilizada em ambulatórios especializados geridos pelos municípios, Estado, Consórcios Intermunicipais de Saúde, hospitais gerais e especializados, serviços contratualizados e unidades de apoio de diagnose e terapia .

Frente à pandemia, esses serviços de saúde, de forma geral, precisaram ter reorganizados os processos de trabalho, a fim de garantir o atendimento oportuno e seguro à subpopulação-alvo. Apesar de não ser uma orientação da SESA-PR o cancelamento de todas as consultas e exames especializados, alguns serviços decidiram suspender suas atividades logo após o diagnóstico do primeiro caso no Estado, em março de 2020. Observada a necessidade de orientar e apoiar as RAS do Paraná no processo organizativo para o enfrentamento a pandemia, a Secretaria, por meio das equipes técnicas, elaborou um plano de contingência, resoluções, notas orientativas e fluxos para atendimentos, apresentados e discutidos a seguir, como corpo deste relato de experiência.

\section{RESOLUÇÕES}

Na Resolução SESA n 338/2020, publicada em março de 2020, ficou estabelecido, em âmbito estadual, a implementação de medidas de enfrentamento da emergência em saúde pública. Foi um marco importante em relação às orientações aos serviços de saúde em todos os níveis de atenção do Estado?.

Em relação ao atendimento ambulatorial, no Art. 23 orienta-se o cancelamento imediato de todas as agendas iniciais de especialidades, com exceção de Cardiologia, Nefrologia, Oncologia (todas as subespecialidades) e Gestação de alto risco' . 
Para garantir as medidas de prevenção e segurança aos usuários e profissionais, foi orientado que os atendimentos devem ser programados de forma a evitar acúmulo de pacientes em horários simultâneos, favorecendo as medidas de controle de contágio potencial ${ }^{9}$. Em relação aos usuários em acompanhamento pelo seguimento especializado, recomenda-se o remanejamento das agendas de retorno, a critério de cada serviço, atendendo à gravidade de cada paciente e priorizando, excepcionalmente, os casos de maior risco terapêutico ${ }^{9}$.

Nos exames e tratamentos complementares especializados, com exceção da radioterapia, quimioterapia e hemodiálise, é preconizada redução mínima de $50 \%$ da agenda, associada à reorganização compulsória do serviço, para evitar acúmulo de pacientes em horários simultâneos.

O transporte sanitário de usuários para atendimentos eletivos também é abordado, com a recomendação de suspensão do serviço nesses casos, e garantia da manutenção do deslocamento para tratamento de alta complexidade, hemodiálise, gestação de alto risco, ou conforme critério das Secretarias Municipais de Saúde, desde que respeitadas as medidas de precaução anteriormente orientadas 9 .

A Resolução SESA n 632/2020, publicada em maio, estabeleceu medidas complementares de controle sanitário a serem implementadas em locais de uso público e coletivo, tais como espaços de natureza comercial, hoteleira, cultural, esportiva, financeira, turística, recreativa, social, religiosa, educacional, industrial, e de saúde, além daqueles administrados por entidades da administração pública direta ou indireta, e prestadores de serviços públicos destinados ao público em geral ${ }^{10}$.

Nesse documento está estabelecido que as principais medidas para o enfrentamento ao coronavírus correspondem ao distanciamento mínimo necessário entre pessoas, de dois metros; higienização das mãos; limpeza e desinfecção de superfícies e ambientes internos e externos; uso de máscara pela população em geral, em espaços de uso público e coletivo; comunicação e orientações 
gerais de prevenção direcionadas à população; além de outras medidas que reforçaram o combate à evolução da doença no Paraná10.

\section{Notas orientativas}

As equipes técnicas da Diretoria de Atenção e Vigilância em Saúde da SESA-PR elaboraram notas orientativas voltadas a grupos prioritários e prestadores de serviços específicos. Até o dia 12 de julho de 2020 haviam sido publicadas 41 notas orientativas, sendo que dez abordam questões relacionadas ao atendimento em ambulatórios especializados em saúde. Os conteúdos dessas nortearam os serviços de saúde nesse nível de atenção, de forma a garantir que usuários com necessidades caracterizadas como essenciais, estratificados pela APS e pertencentes a grupos prioritários de acompanhamento, não tivessem seu direito de acesso à saúde cerceado nesse período.

A primeira nota orientativa com abordagem da organização do atendimento aos idosos frente à pandemia em rede $\left(n^{\circ}\right.$ 04/2020) foi publicada em março de 2020 e recomenda a suspensão dos atendimentos ambulatoriais presenciais a idosos pertencentes à subpopulação-alvo dos ambulatórios. Entretanto, em relação à equipe especializada, é orientado que a mesma deve permanecer exercendo seu papel de apoio à APS, através de discussões de casos, teleconsultas e matriciamentos ${ }^{11}$.

A linha de cuidado materno infantil foi tema da nota orientativa $\mathrm{n}^{\circ}$ 09/2020, publicada ainda em março, que abordou o atendimento às gestantes na RAS no período pandêmico e, em especial, nos ambulatórios. A principal orientação a ser observada nesse nível de atenção foi o seguimento presencial das gestantes estratificadas como risco intermediário e alto risco, com comorbidades associadas, garantindo as medidas de precaução preconizadas, a fim de diminuir o risco de contaminações pelo SARS-CoV- $2^{12}$.

No atendimento em puericultura, conforme a nota orientativa $n^{\circ} 29 / 2020$, orienta-se que crianças de alto risco devem ser acompanhadas na APS, e compartilhadas com as equipes da $A A E$, preferencialmente, na modalidade presen- 
cial, quando não for possível, o apoio técnico poderá se dar por telefone ${ }^{13}$.

Na saúde mental, os serviços ambulatoriais que realizam a assistência dessa subpopulação-alvo são orientados a garantir o acompanhamento dos usuários em atividades caracterizadas como essenciais, entre elas, o manejo da crise; o acolhimento às demandas de agudização de casos novos, ou já acompanhados; emissões de atestados e renovações de prescrições médicas; e monitoramento dos usuários com baixa adesão ao plano terapêutico ${ }^{14}$.

A nota orientativa $n^{\circ}$ 18/2020 salienta a organização dos atendimentos em oncologia no período pandêmico. Em relação às consultas de acompanhamento e controle do tratamento, é recomendada a avaliação da possibilidade de adiamento dos atendimentos. Nos casos em que a consulta presencial for necessária, orienta-se a reorganização dos serviços, através da garantia de medidas de proteção para pacientes e profissionais ${ }^{15}$.

Nas terapias adjuvantes, como quimioterapia e radioterapia, é preconizada a implementação de práticas com o intuito de reduzir a disseminação de patógenos respiratórios durante os procedimentos, entre elas, a realização de triagem prévia de sintomático respiratório; uso de máscara de proteção; higienização correta das mãos; e demais medidas de proteção aplicadas à comunidade ${ }^{15}$.

Nos serviços de diálise, a nota orientativa $n^{\circ}$ 27/2020 deixa clara a correta organização dos fluxos e espaços físicos, tendo em vista que esses serviços são essenciais e não podem reduzir a oferta, e nem o tempo de duração das terapias. De acordo com a nota, os pacientes que apresentarem sintomas gripais devem comunicar previamente, por telefone, para que o serviço de diálise possa organizar a recepção e a terapia segura para pacientes e profissionais ${ }^{16}$.

Em atendimentos à pacientes dialíticos, suspeitos ou confirmados para Covid-19, recomenda-se local exclusivo para a sua permanência, onde esse deve usar máscara cirúrgica e higienizar oportunamente as mãos. Quando o serviço possuir mais de um paciente suspeito ou confirmado, deverá realizar o isolamento por coorte, empregando o uso de barreiras físicas e o distanciamento 
mínimo recomendado ${ }^{16}$.

Em relação ao transporte sanitário, a nota orientativa n²0/2020, publicada em abril, determina a manutenção do translado de pacientes apenas nos casos de urgência e emergência, para a manutenção de tratamentos de alta complexidade (como quimioterapia e hemodiálise), nos atendimentos às gestantes de alto risco e a critério das Secretarias Municipais de Saúde ${ }^{17}$. Para garantir a segurança dos pacientes transportados, recomenda-se evitar aglomerações para reduzir a possibilidade de contaminação, com veículos operando com uma capacidade de lotação reduzida a 50\%. Além disso, determina medidas adicionais, como abertura das janelas para circulação do ar, evitar o uso de ar-condicionado, e dispor de dispensadores de álcool em gel ${ }^{17}$.

Sobre o atendimento aos pacientes com Hipertensão Arterial Sistêmica e Diabetes Mellitus nos ambulatórios especializados, a nota orientativa n 32/2020 salienta a importância de a equipe especializada conhecer sua subpopulação alvo em acompanhamento, para identificar a necessidade de atendimento presencial. O monitoramento através de ligações telefônicas, de aplicativos de mensagens ou mídias digitais também está incluído como alternativa de atendimento ${ }^{18}$. Aos usuários da APS, recentemente estratificados, e com necessidade de atendimento especializado, é recomendado o compartilhamento do cuidado, através do apoio da equipe dos ambulatórios aos profissionais, envolvendo ações de matriciamento, supervisão do especialista e até atendimentos presenciais, quando verificada a necessidade pelas equipes ${ }^{18}$.

Uma nota específica foi elaborada para nortear as ações nos ambulatórios multiprofissionais organizados no Modelo de Atenção às Condições Crônicas (MACC), geridos pelos consórcios intermunicipais de saúde, que atendem nas linhas de cuidado prioritárias para o Estado: Materno Infantil, Idoso, Hipertensão e Diabetes, e Saúde Mental ${ }^{19}$.

A referida nota de $n^{\circ} 33 / 2020$, publicada em maio, aborda a importância do acompanhamento das condições crônicas no período pandêmico, através de ações 
articuladas dentro da RAS e, em especial, a reorganização dos processos de trabaIho da $A A E$, para continuidade dos atendimentos, quer seja presencial, ou não ${ }^{19}$.

As principais questões elencadas referem-se às recomendações para o manejo das condições crônicas nesse período, evidenciando a importância da estratificação de risco, como direção às ações das equipes multiprofissionais; quais condutas a serem tomadas pela APS e AAE frente aos novos usuários que necessitam de encaminhamento; os cuidados a serem observados nos atendimentos presenciais; e como monitorar a subpopulação-alvo sem instabilidade clínica de forma remota ${ }^{19}$.

Ao início de julho de 2020 foi publicada a nota orientativa $n^{\circ} 39 / 2020$, com orientações referentes ao atendimento odontológico nos serviços públicos frente à Covid-19. O documento deixa explícito a organização dos atendimentos na APS, nos Centros de Especialidades Odontológicas e nos Laboratórios de Regionais de Prótese Dentária ${ }^{20}$. O conteúdo ressalta que a SESA-PR não se opõe ao retorno dos atendimentos odontológicos eletivos, desde que observadas as recomendações em relação aos cuidados durante o atendimento, proporcionando segurança ao paciente e profissional, com atenção ao processamento dos materiais, a fim de mitigar o risco de transmissão viral20.

\section{Plano de contingência}

O plano de contingência é um documento que sistematiza as etapas de preparação de uma instituição para o enfrentamento de determinado evento, considerado extraordinário até o momento. Na saúde pública, a estratégia é, usualmente, utilizada para prever planos de proteção, prevenção e respostas rápidas às ameaças identificadas, integrando esforços das instituições envolvidas. No documento são previstos os riscos e quais serão as ações executadas para combatê-los, caso venham a se concretizar ${ }^{21}$.

É recomendada a sua construção por todos os atores envolvidos no processo de enfrentamento a determinado agravo, independentemente se atuante na assistência ou na gestão. Na pandemia ocasionada pelo coronavírus, 
a Organização Mundial da Saúde, Ministério da Saúde, Secretarias Estaduais e Municipais de Saúde utilizaram-se dessa ferramenta para organizar o plano de respostas às demandas decorrentes da pandemia por SARS-CoV-221.

No Paraná, um plano foi construído ainda no mês de abril pelo Centro de Operações de Emergências (COE), com o intuito de auxiliar o Estado na resposta ao enfrentamento à Covid-19, e definir responsabilidades, integralidade nas ações de prevenção e monitoramento, bem como congruência na assistência à saúde da população, independentemente do nível de complexidade ${ }^{22}$.

A organização das respostas foi descrita por níveis crescentes de ações, baseados nos cenários epidemiológicos esperados. No nível 1, denominado de Atenção, as ações descritas correspondem a um cenário com a presença de apenas casos suspeitos de Covid-19. O nível 2, caracterizado como um cenário de Ameaça, corresponde ao período no qual casos suspeitos tornam-se confirmados laboratorialmente. E por fim o nível 3, de Execução, é caracterizado pela confirmação de transmissão local do primeiro caso do Covid-1922.

A APS, rede de urgência e emergência, e atenção hospitalar foram os níveis de complexidade mais citados no plano de contingência, com atribuições específicas nesses pontos de atenção, conforme a evolução do número de casos suspeitos e confirmados. Em junho de 2020 o documento sofreu atualizações, com publicação de nova versão, considerando o momento epidemiológico que o Estado vivencia.

Na versão atualizada, a AAE foi contemplada, principalmente, nas ações da assistência à saúde relativas ao acompanhamento e monitoramento dos usuários com condições crônicas e instabilidade clínica persistente, pertencentes à subpopulação-alvo dos ambulatórios. Outras orientações foram inseridas no documento, como a instrução a esses serviços em relação à importância da notificação imediata de casos suspeitos; a suspensão de atendimentos eletivos (excetuando-se os atendimentos essenciais, tais como gestantes de alto risco, cardiologia, nefrologia e oncologia e os com instabilidade clínica); e o remaneja- 
mento de agendas de usuários em acompanhamento especializado ${ }^{23}$.

\section{CONSIDERAÇÕES FINAIS}

Por tratar-se de uma doença viral com evidências científicas pouco concretas em relação ao seu comportamento, e considerando o seu alto poder de propagação, essa exigiu a reorganização dos sistemas de saúde em nível mundial, com foco no atendimento aos casos agudos e sintomáticos da Covid-19. Entretanto, além dessa recente demanda trazida pela pandemia, os serviços de saúde precisam equilibrar os esforços para garantir que as demais condições de saúde que acometem a população também sejam contempladas nos planejamentos e planos de intervenção.

Foi na perspectiva da continuidade das ações na RAS, de forma resolutiva e segura, que os gestores e profissionais de saúde em todos os níveis de atenção precisaram direcionar seus esforços. A SESA-PR tem trabalhado de forma incessante, a fim de garantir que os serviços de saúde permaneçam realizando o seu papel, independente do nível de complexidade, de forma presencial ou remota, assegurando o direto de acesso à saúde de qualidade a todos os paranaenses.

\section{AGRADECIMENTOS}

Agradeço à Elaine Drehmer de Almeida Cruz, apoiadora pela OPAS junto à SESA-PR, pelas contribuições ao texto.

\section{REFERÊNCIAS}

1. Organização Pan-Americana da Saúde (OPAS). Folha informativa - COVID-19 (doença causada pelo novo coronavírus) [Internet]. Brasília: 2020. [citado em 2020 Mai 27]. Disponível em: https://www.paho.org/bra/index.php?option=com_ content\&view=article\&id=6101:covid19\&ltemid=875.

2. Conselho Nacional dos Secretários de Saúde (CONASS). COVID-19: Guia orientador para o enfrentamento da pandemia na Rede de Atenção à Saúde [Internet]. Brasília: CONASS; 2020. [citado em 2020 Mai 27]. Disponível em: https://www. conasems.org.br/wp-content/uploads/2020/05/Instrumento-Orientador-Conass-Conasems-VERS\%C3\%830-FINAL-3.pdf. 
3. Brasil. Constituição, 1988. Constituição da República Federativa do Brasil. Brasília: Senado Federal; 1988. [citado em 2020 Mai 27]. Disponível em: http://conselho.saude.gov.br/web_sus20anos/20anossus/legislacao/constituicaofederal.pdf.

4. Mendes EV. As Redes de Atenção à Saúde. Brasil: Organização Pan-Americana da Saúde; 2011. [citado em 2020 Mai 27 ]. Disponível em: http://bvsms.saude.gov.br/bvs/publicacoes/redes_de_atencao_saude.pdf.

5. Canzian F. Atenção básica vê 'terceira onda' de doentes atingindo o sistema de saúde. Folha de São Paulo; 2020 abr 26. Saúde. [citado em 2020 Mai 27]. Disponível em: https://www1.folha.uol.com.br/equilibrioesaude/2020/04/atencao-basicave-terceira-onda-de-doentes-atingindo-o-sistema-de-saude.shtml.

6. Queiroga M. Na pandemia, a terceira onda de mortalidade será por doenças crônicas. Associação Nacional de Hospitais Privados (ANHP). Sociedade Brasileira de Cardiologia; 2020 jun. COVID-19 artigos. [citado em 2020 Mai 30]. Disponível em: https://www.anahp.com.br/wp-content/uploads/2020/06/artigo-SBC.pdf.

7. Solla J, Chioro A. Atenção Ambulatorial Especializada: Políticas e Sistemas de Saúde no Brasil. Escola de Saúde do Paraná. [citado em 2020 Mai 30]. Disponível em: http://www.escoladesaude.pr.gov.br/arquivos/File/ATENCAO_AMBULATORIAL_ ESPECIALIZADA_Solla_e_Chioro.pdf.

8. Cadastro Nacional de Estabelecimentos de Saúde (CNES). Relatórios: tipos de estabelecimentos no Paraná. Paraná: Secretaria de Atenção à Saúde; 2020. [citado em 2020 Mai 30]. Disponível em: http://cnes2.datasus.gov.br/Mod_Ind_ Unidade.asp?VEstado=41\&VMun=\&VComp=00\&VUni $=$.

9. Paraná. Resolução SESA n 338/2020. Implementação de medidas de enfrentamento da emergência em saúde pública de importância nacional e internacional decorrente do Coronavírus - COVID-2019. Secretaria de Estado de Saúde do Paraná; 2020. [citado em 2020 Mai 30]. Disponível em: http://www.saude.pr.gov.br/sites/default/arquivos_restritos/files/ documento/2020-04/338_20.pdf.

10. Paraná. Resolução SESA n 632/2020. Dispõe sobre medidas complementares de controle sanitário a serem adotadas para enfrentamento da COVID-19. 2020. Secretaria de Estado de Saúde do Paraná; 2020. [citado em 2020 Mai 30]. Disponível em: http://www.saude.pr.gov.br/sites/default/arquivos_restritos/files/documento/2020-06/632_20.pdf.

11. Secretaria de Estado de Saúde do Paraná (SESA PR). Nota Orientativa nº 04 - Atendimentos dos idosos frente à pandemia COVID-19 [Internet]. Paraná; 2020. [citado em 2020 Mai 30]. Disponível em: http://www.saude.pr.gov.br/sites/default/ arquivos_restritos/files/documento/2020-06/no_04_atendimento_dos_idosos_frente_a_pandemia_covid_19.pdf.

12. Secretaria de Estado de Saúde do Paraná (SESA PR). Nota Orientativa $n^{\circ} 09$ - Orientações às equipes e profissionais sobre linha de cuidado materno infantil durante a emergência em saúde pública coronavírus covid-19 [Internet]. Paraná; 2020. [citado em 2020 Jun 5]. Disponível em: http://www.saude.pr.gov.br/sites/default/arquivos_restritos/files/ documento/2020-06/no_09_linha_de_cuidado_materno_infantil_v2.pdf.

13. Secretaria de Estado de Saúde do Paraná (SESA PR). Nota Orientativa nº 29 - Atendimento em puericultura [Internet]. Paraná: SESA; 2020 [citado em 2020 Jun 5]. Disponível em: http://www.saude.pr.gov.br/sites/default/arquivos_restritos/files/ documento/2020-06/no_29_atendimento_em_puericultura.pdf.

14. Secretaria de Estado de Saúde do Paraná (SESA PR). Nota Orientativa nº 15 - Saúde Mental [Internet]. Paraná: SESA; 2020. [citado em 2020 Jun 5]. Disponível em: http://www.saude.pr.gov.br/sites/default/arquivos_restritos/files/ documento/2020-06/no_15_saude_mental_v2.pdf.

15. Secretaria de Estado de Saúde do Paraná (SESA PR). Nota Orientativa $n^{\circ} 18$ - Atendimentos em oncologia frente a pandemia COVID-19 [Internet]. Paraná: SESA; 2020. [citado em 2020 Jun 5]. Disponível em: http://www.saude.pr.gov.br/sites/ default/arquivos_restritos/files/documento/2020-06/no_18_atendimentos_em_oncologia_frente_a_pandemia_covid_19.pdf.

16. Secretaria de Estado de Saúde do Paraná (SESA PR). Nota Orientativa nº 27 - Medidas de prevenção e controle da COVID-19 para serviços de diálise [Internet]. Paraná: SESA; 2020. [citado em 2020 Jun 5]. Disponível em: http://www. saude.pr.gov.br/sites/default/arquivos_restritos/files/documento/202006/no_27_medidas_de_prevencao_e_controle_da covid_19_para_servicos_de_dialise_v2.pdf.

17. Secretaria de Estado de Saúde do Paraná (SESA PR). Nota Orientativa n 20 - Orientações gerais para a prevenção da COVID-19 em transporte sanitário [Internet]. Paraná: SESA; 2020. [citado em 2020 Jun 5]. Disponível em: http://www. saude.pr.gov.br/sites/default/arquivos_restritos/files/documento/202006/no_20_orientacoes_gerais_para_prevencao_da_ covid_19_em_transporte_sanitario_0.pdf.

18. Secretaria de Estado de Saúde do Paraná (SESA PR). Nota Orientativa no 32 - Cuidados às pessoas com Hipertensão Arterial Sistêmica e Diabetes Mellitus frente à pandemia da COVID-19 [Internet]. Paraná: SESA; 2020. [citado em 2020 Jun 5]. Disponível em: http://www.saude.pr.gov.br/sites/default/arquivos_restritos/files/documento/2020-06/no_32_cuidados_as_ pessoas_com_hipertensao_arterial_sistemica_e_diabettes_mellitus.pdf.

19. Secretaria de Estado de Saúde do Paraná (SESA PR). Nota Orientativa no 33 - Nota orientativa para os ambulatórios organizados no Modelo de Atenção às Condições Crônicas em tempos de COVID-19 [Internet]. Paraná: SESA; 2020. [citado 
em 2020 Jun 05]. Disponível em: http://www.saude.pr.gov.br/sites/default/arquivos_restritos/files/documento/2020-06/ NO_33_AMBULATORIOS_ORGANIZADOS_NO_MODELO_DE_ATENCAO_AS_CONDICOES_\%20CRONICAS_V6.pdf.

20. Secretaria de Estado de Saúde do Paraná (SESA PR). Nota Orientativa n 39 - Orientações referentes ao atendimento odontológico nos serviços públicos frente a COVID-19 [Internet]. Paraná: SESA; 2020. [citado em 2020 Jun 5]. Disponível em: http://www.saude.pr.gov.br/sites/default/arquivos_restritos/files/documento/2020-07/NO_39_ORIENTACOES_REFERENTES_ AO_ATENDIMENTO_ODONTOLOGICO_NOS_SERV\%20ICOS_PUBLICOS_FRENTE_A_COVID_19_V2.pdf.

21. Agência Nacional de Vigilância Sanitária (ANVISA). Modelo de Plano de Contingência para Emergência de Interesse de Saúde Pública [Internet]. Brasília: Anvisa; 2020. Disponível em: http://portal.anvisa.gov.br/documents/219201/5777769/ Protocolos+e+plano+de+conting\%C3\%AAncia/cfb8e39a-4e0d-4683-ac3e-b7a211998135.

22. Secretaria de Estado de Saúde do Paraná (SESA PR). Plano de Contingência do Paraná COVID-19 [Internet]. Paraná: SESA; 2020. [citado em 2020 Jun 5]. Disponível em: http://www.saude.pr.gov.br/sites/default/arquivos_restritos/files/ documento/2020-04/planocovid19final.pdf.

23. Secretaria de Estado de Saúde do Paraná. Plano de Contingência do Paraná COVID-19 (Nível 3 - Execução) [Internet]. Paraná: SESA; 2020. [citado em 2020 Jun 5]. Disponível em: http://www.saude.pr.gov.br/sites/default/arquivos_restritos/files/ documento/2020-06/plano_de_contingencia_do_parana_covid_19_nivel_3_atualizado.pdf.

\section{RECEBIDO:13/08/2020 \\ ACEITO: $13 / 10 / 2020$}

